\title{
1 idCOV: a pipeline for quick clade identification of
}

\section{SARS-CoV-2 isolates}

3 Xun Zhu ${ }^{1, \dagger}$, Ti-Cheng Chang ${ }^{1, \dagger}$, Richard $\mathrm{Webby}^{2}$, and Gang $\mathrm{Wu}^{1, *}$

$4 \quad{ }^{1}$ Center for Applied Bioinformatics, St. Jude Children's Research Hospital. 262 Danny Thomas Pl,

$5 \quad$ Memphis, TN 38105

$6 \quad{ }^{2}$ Department of Infectious Disease, St. Jude Children's Research Hospital. 262 Danny Thomas Pl, Mem-

7 phis, TN 38105

$8 \quad$ * To whom correspondence should be addressed.

$9{ }^{\dagger}$ The authors wish it to be known that these authors contributed equally.

\section{Abstract}

12 idCOV is a phylogenetic pipeline for quickly identifying the clades of SARS-CoV-2 virus isolates from

13 raw sequencing data based on a selected clade-defining marker list. Using a public dataset, we show that

14 idCOV can make equivalent calls as annotated by Nextstrain.org on all three common clade systems us-

15 ing user uploaded FastQ files directly. Web and equivalent command-line interfaces are available. It can

16 be deployed on any Linux environment, including personal computer, HPC and the cloud. The source

17 code is available at https://github.com/xz-stjude/idcov. A documentation for installation can be found at

18 https://github.com/xz-stjude/idcov/blob/master/README.md.

\section{Introduction}

20 The on-going Coronavirus disease 2019 (Covid-19) pandemic has resulted in over 734,000 deaths, affect-

21 ing more than 188 countries and territories (CSSE, 2020; Dong, et al., 2020). The general impact of the

22 disease calls for a quick response from the local health system at every stage of its transmission. 
Severe acute respiratory syndrome coronavirus 2 (SARS-CoV-2; previously known as 2019-nCoV) is the pathogenic cause of Covid-19 (Lescure, et al., 2020). Quickly identifying the genetic clade of the virus helps develop an understanding of the local transmission of the disease. The recent NYC study (Gonzalez-Reiche, et al., 2020), for example, identified crucial local transmission events, plurality of virus introductions, and the possible sources of these introductions. The classification of the infected virus can also provide more granular information to contact tracing and for epidemiological studies to understand disease severity and host genetic susceptibility to different lineages of SARS-CoV-2.

In order to quickly identify the clade of an isolate of SARS-Cov-2 given its sequencing FastQ files, we have developed a bioinformatics pipeline and a user-friendly web interface. An equivalent commandline interface is also supplemented for batch-processing many samples in a terminal environment. We introduce this system as $i d C O V$.

\section{Pipeline}

\section{Input}

The pipeline (Figure 1) starts with the sequencing data derived from SARS-Cov-2 isolates in the format of pair-end FastQ files. These files are either available on the server or uploaded by the user through the web or the command-line interface. The fragments in the FastQ files are then aligned to a reference genome.

\section{Alignment}

Normally a virus reference genome is used for alignment. However, since sequencing library preparations might be generated from clinical material, some of the sequencing reads in the FastQ files may be from the human host. To account for this, we aggregated the Wuhan-Hu-1 genome (Wu, et al., 2020) with the GRCh38 human genome and used the combined genome as the reference for the alignment. The alignment is done using BWA, version 0.7.17 ( $\mathrm{Li}$ and Durbin, 2009). The "bwa mem" algorithm is used by default. After the alignment, the result BAM files are first sorted by the fragment locations and then in- 
dexed, both using the SAMtools software, version 1.10 (Li, et al., 2009). This post-processing is to improve the performance of subsequent steps.

\section{Mutations Calculation}

The virus reference genome (Wuhan-Hu-1) is seen as the original genome and any deviation from it is seen as a mutation. We run FreeBayes, version 1.3.2 (Garrison and Marth, 2012) on the BAM files generated above and the reference genome to calculate the mutations. The ploidy is set to one and the fragments mapping to the human genome are removed in the input to FreeBayes. The default values are used for the rest of the FreeBayes parameters. The output from FreeBayes is a list of confident mutations stored in a VCF file.

\section{Partial genome reconstruction}

Depending on the depth and distribution of the sequencing, there might be regions in the viral genome to which no fragments were mapped. In the VCF output from FreeBayes, an absence of mutations due to unmapped regions must be distinguished from an actual absence of mutations (as deemed by FreeBayes.)

Therefore, before we compare the markers, we reconstruct the partial genome of the isolate by masking the un-mapped regions in the reference gnome, and then apply the mutations as called by FreeBayes to the masked genome.

\section{Isolate identification}

A marker profile is generated from the reconstructed genome. The marker profile consists of the base pairs at a list of predefined marker locations. These marker locations are dictated by the clade system. Currently, three clade systems are used in the classification step: the old Nextstrain, the new Nextstrain (Hodcroft, et al., 2020), and the GISAID clade system (e.V., 2020).

Finally, given a clade system, the marker profile is compared with the reference clade-defining marker profiles (Supplementary Table 1). If the marker profile of the isolate is the same as that of a designated clade, the isolate is called as the said clade. If the marker profile of the isolate is different from that of any of the clades, the clade with a marker profile most similar (in terms of Manhattan distance) is 
reported, along with the Manhattan distance between the two. When multiple clades are tied as having the least Manhattan distance, the clade of the isolate is considered ambiguous between the tied clades. If none of the Manhattan distances is below 4.5, the clade of the isolate is considered undetermined.

For two marker profiles $A=\left(a_{1}, a_{2}, \ldots, a_{n}\right)$ and $B=\left(b_{1}, b_{2}, \ldots, b_{n}\right)$, their Manhattan distance is defined as $D_{M}(A, B)=\sum_{i=1}^{n} M_{i}$, where $M_{i}=0$ when $a_{i}=b_{i}, M_{i}=1$ when $a_{i} \neq b_{i}$, and $M_{i}=0.5$ when either $a_{i}$ or $b_{i}$ is undetermined due to lack of mapped reads at the marker region.

\section{Software}

\section{The web user interface}

After idCOV is deployed on a Linux environment, it can be accessed using any modern browser. A user can either upload the gzipped FastQ files stored on the client machine or specify a list of gzipped FastQ files already on the server file pool. Each pair of gzipped FastQ files needs to be named "*R1.fastq.gz" and "*R2.fastq.gz" so that their parity can be determined. A project can be created with the uploaded or specified files. The pipeline can be started by running a project. The sessions are tracked on the server, so the progress of any running project will not be lost when the client browser is closed. (Supplementary Figure 1)

\section{The command line interface}

A command line interface will also be provided on the server-side after idCOV is deployed. Assuming terminal access (either direct or through SSH), a user can use the command "idcov" in the command line to run the pipeline directly on any gzipped FastQ files on the server.

\section{Data security}

When deployed on a private computer or a cloud VM, all uploaded data is stored in a folder specified by a Docker volume binding parameter. The administrator of the computer can set the appropriate access permissions for this folder to ensure the data security. 


\section{Implementation}

The workflow management is done by using the Nextflow system (Di Tommaso, et al., 2017). The individual steps are broken down into Nextflow processes, and the deployment on multiple platforms is done by preparing a configuration for HPC and another for cloud environment. The latter can also be used on personal computers. A Datahike database (Kühne, et al., 2020) is used for storing user accounts, sessions, and job tracking. The server is written in Clojure using the Ring library. The web frontend is written in ClojureScript using the Fulcro library. The command-line interface is written in Python.

\section{Testing}

The Clojure/ClojureScript code is unit-tested using the clojure.test library. The web UI is tested in Google Chrome Version 84.0.4147.105 (Official Build) (64-bit) and Mozilla Firefox Version 79.0.

We tested the entire workflow on 18 representative samples from a SARS-Cov-2 genomic sequencing dataset reported by the Peter Doherty Institute for Infection and Immunity and the Victorian Department of Health and Human Services in Australia (Seemann, et al., 2020). These samples included viral sequences representative of L, V, S, G, GR, and GH clades (GISAID clade nomenclature). idCOV correctly identified the clade of all viruses, regardless of the clade-defining marker profile used (i.e., Nextstrain old, new, and GISAID) (Figure 3). The full report is attached in the Supplementary Materials.

\section{Conclusion}

Rapid classification of SARS-Cov-2 viruses into their respective genetic clades helps characterize transmission patterns and assess local and global epidemiologic trends. idCOV provides an easy portal to a complete bioinformatics pipeline that identifies a virus' clade using the three most widely used clade systems. The system is internally tested and has been shown to provide valuable insights to clinicians and biologists in a timely manner.

\section{Funding}


118

119

120

\section{Conflict of Interest}

122 None declared.

This research was supported in part by American Lebanese Syrian Associated Charities (ALSAC) and the National Cancer Institute grant P30 CA021765. The content is solely the responsibility of the authors and does not necessarily represent the official views of the National Institutes of Health.

\section{Figures}

Figure 1: The bioinformatics pipeline of idCOV.

Figure 2: idCOV user experience.

Figure 3: Screenshots of an example report of idCOV. The dataset consists of a random selection of isolates from Seemann, et al., 2020 covering all major clades. Table A) shows the final calls with color scheme consistent with Nextstrain.org. Table B) shows the Manhattan distances between every sample and every clade. A smaller Manhattan distance indicates a higher degree of similarity. Table C) shows the marker profiles and Table D) shows the marker coverages. When a marker is covered by fewer than 10 reads, the marker is deemed as undetermined and denoted as a question mark (?).

\section{References}

CSSE. COVID-19 Dashboard by the Center for Systems Science and Engineering (CSSE) at Johns

134 Hopkins University (JHU). In. ArcGIS Online (arcgis.com); 2020.

135 Di Tommaso, P., et al. Nextflow enables reproducible computational workflows. Nature biotechnology

$136 \quad 2017 ; 35(4): 316-319$.

137 Dong, E., Du, H. and Gardner, L. An interactive web-based dashboard to track COVID-19 in real time.

138 The Lancet infectious diseases 2020;20(5):533-534.

139 e.V., F.v.G. GISAID - Clade and lineage nomenclature aids in genomic epidemiology of active hCoV-19

140 viruses. In. Gisaid.org: GISAID; 2020. 
141 Garrison, E. and Marth, G. Haplotype-based variant detection from short-read sequencing. arXiv preprint

142 arXiv:1207.3907 2012.

143 Gonzalez-Reiche, A.S., et al. Introductions and early spread of SARS-CoV-2 in the New York City area.

$144 \quad$ Science 2020.

145 Hodcroft, E.B., et al. Year-letter Genetic Clade Naming for SARS-CoV-2 on Nextstain.org. In.

146 Nextstrain.org: Nextstrain; 2020.

147 Kühne, K., Weilbach, C. and Prokopov, N. Datahike is a durable Datalog database powered by an

148 efficient Datalog query engine. In. Github.com; 2020.

149 Lescure, F.-X., et al. Clinical and virological data of the first cases of COVID-19 in Europe: a case series.

150 The Lancet Infectious Diseases 2020.

$151 \mathrm{Li}, \mathrm{H}$. and Durbin, R. Fast and accurate short read alignment with Burrows-Wheeler transform.

152 bioinformatics 2009;25(14):1754-1760.

153 Li, H., et al. The sequence alignment/map format and SAMtools. Bioinformatics 2009;25(16):2078-2079.

154 Seemann, T., et al. Tracking the COVID-19 pandemic in Australia using genomics. medRxiv 2020.

155 Wu, F., et al. A new coronavirus associated with human respiratory disease in China. Nature

$156 \quad 2020 ; 579(7798): 265-269$.

157 


$$
\overline{\bar{E}} \overline{\underline{ }}
$$
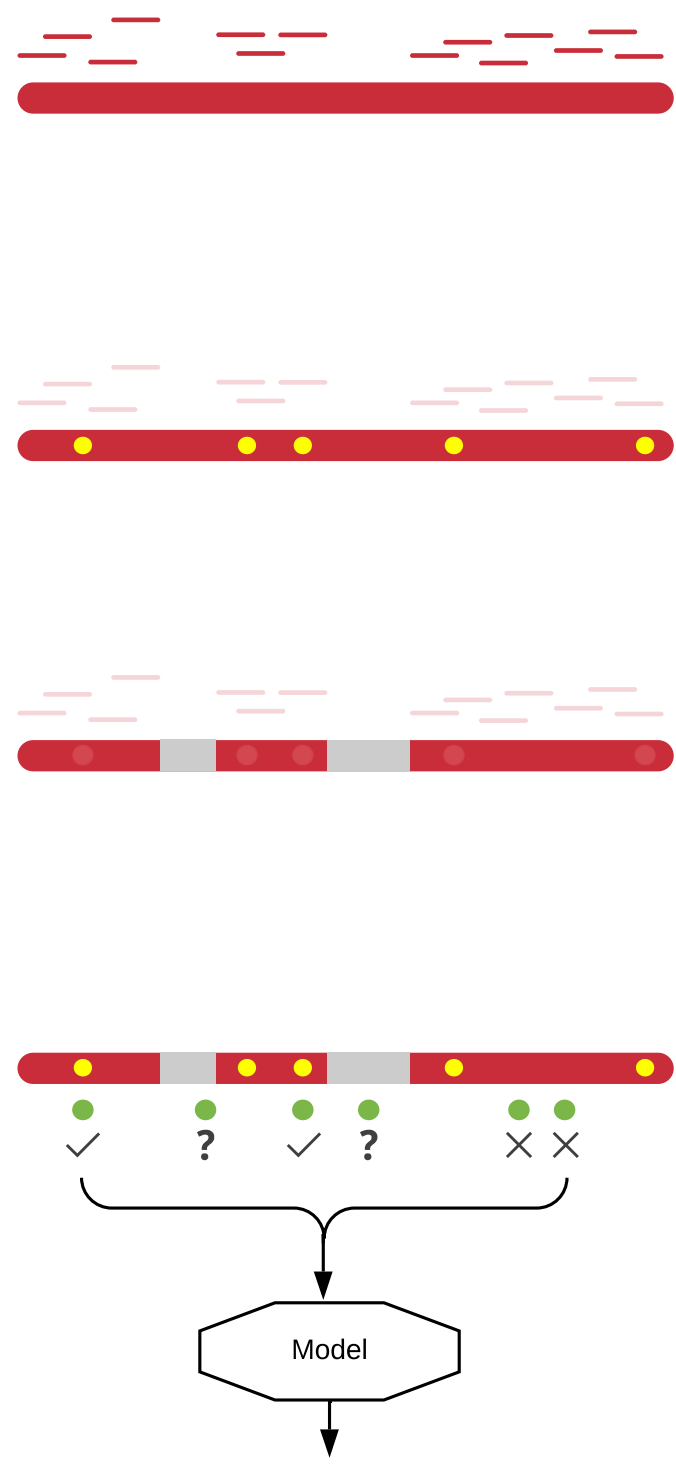

Class (strain)
User-submitted files (FastQ)

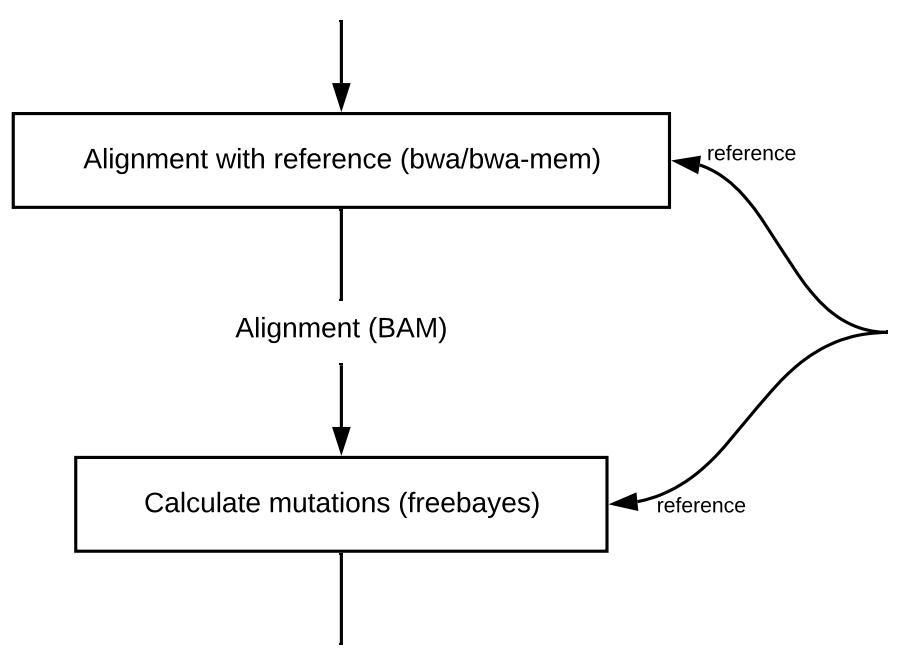

the original Wuhan strain + hg38 (FastA)

List of confident mutations (VCF)

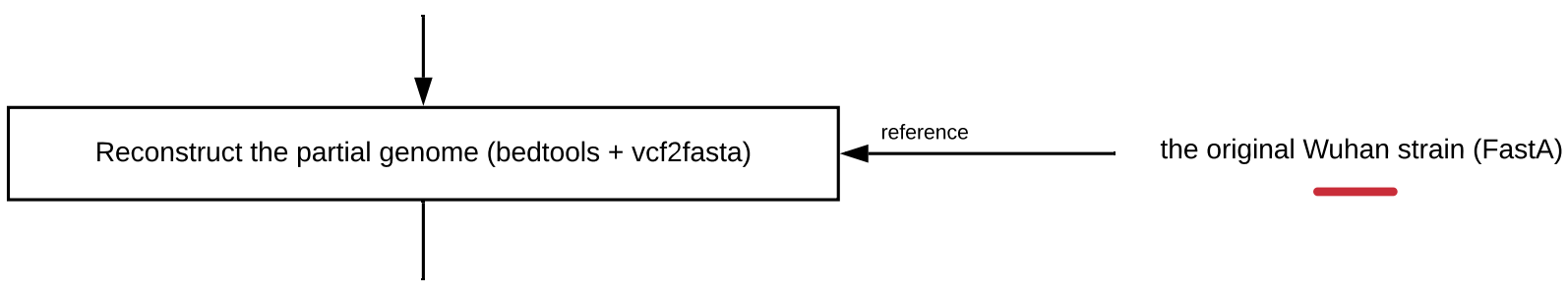

Sample genome with uncovered regions masked (FastA)

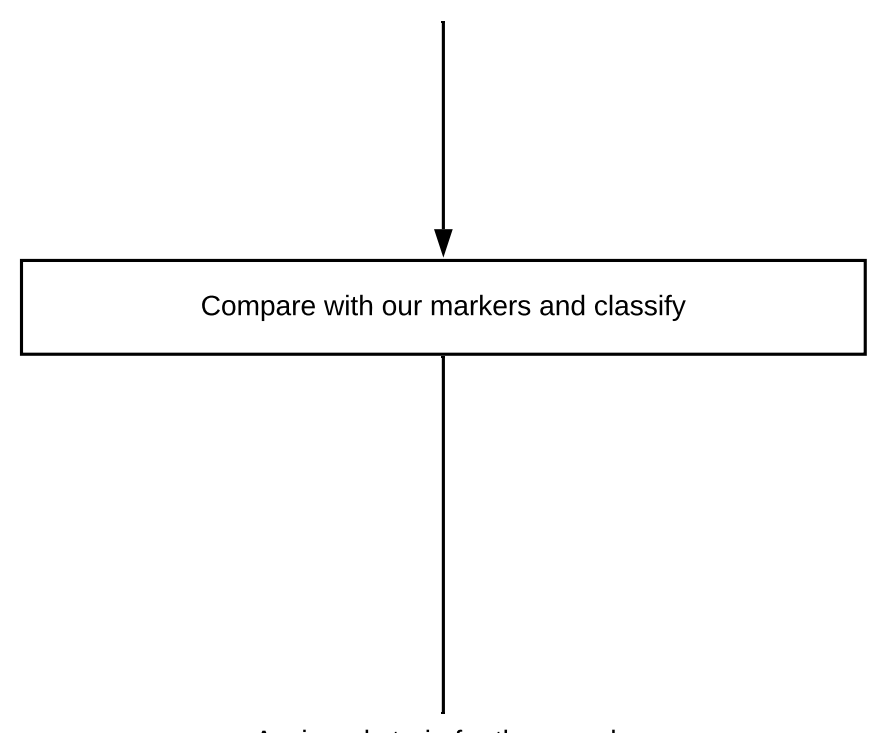

Assigned strain for the sample 


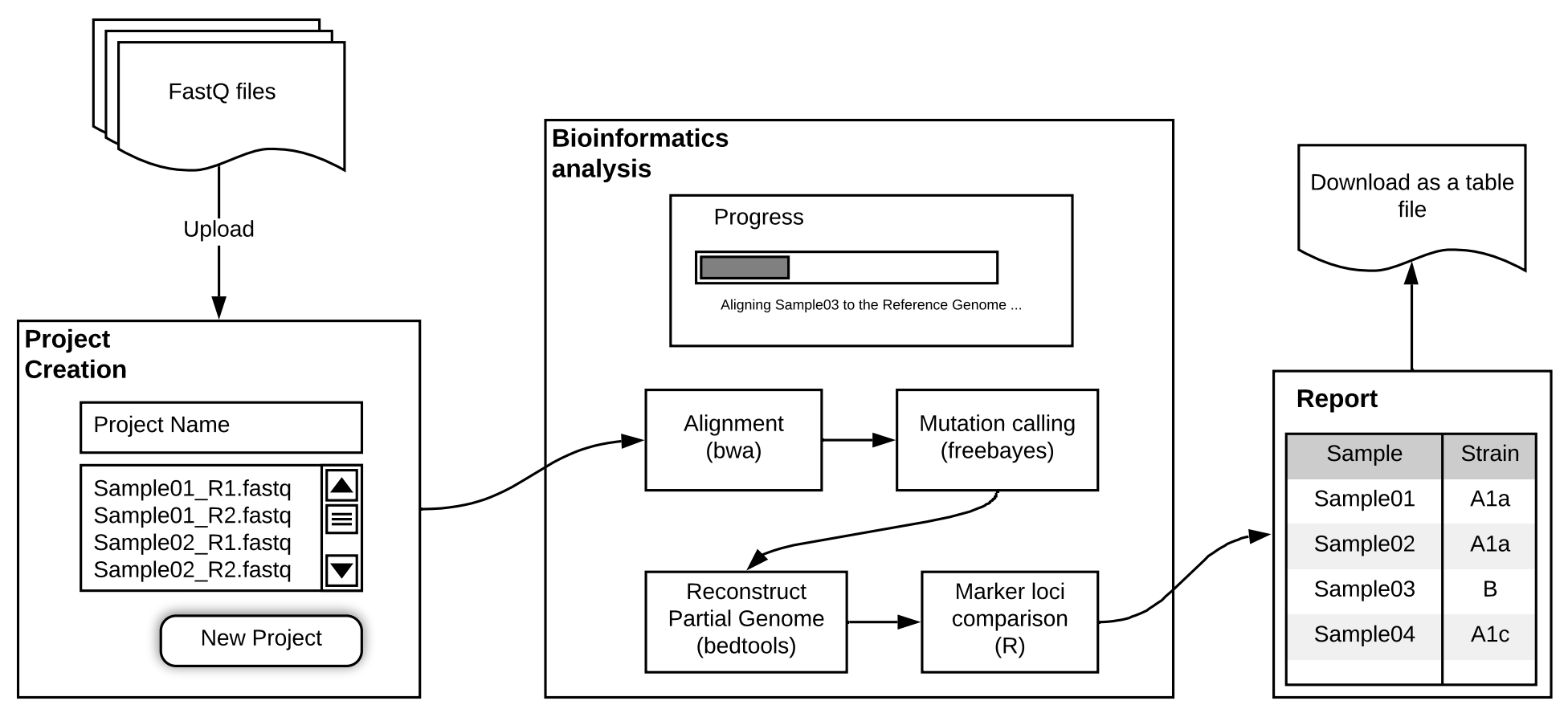




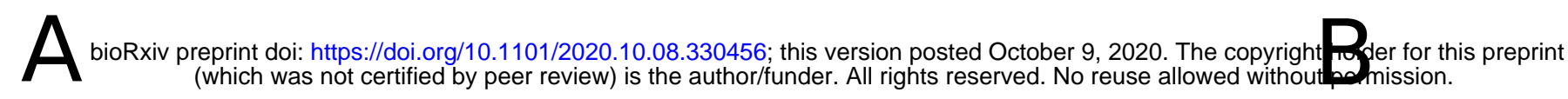

Calls

\begin{tabular}{|c|c|c|c|}
\hline Sample ID & GISAID & New Nextstrain & Old Nextstrain \\
\hline TAS111 & GH_1 & 200 & A2 \\
\hline TAS221 & $\mathrm{s} \__{-} 2$ & 198_2 & B1 \\
\hline TAS255 & $s_{-} 1$ & 198__1 & B/B4 \\
\hline TAS78 & 01 & $19 A_{2}$ & A1a \\
\hline TAS87 & $s_{-1}$ & 198_ 1 & B) 84 \\
\hline Vicor & $\mathrm{s}_{-1} 1$ & 198_1 & B/84 \\
\hline VIC146 & 01 & 19A 2 & A1a \\
\hline VIC147 & $\mathrm{GH}_{-1}$ & 200 & A2 \\
\hline VIC149 & G & 20A_1 & 2 \\
\hline VIC1490 & {$\left[/ 0-1 / 0 \_2 / \sqrt{2}\right.$} & 19A 1 & A3 \\
\hline VIC1783 & GH_2 & $20 A_{2} 2$ & 2 \\
\hline VIC2284 & $G R$ & 208 & A2a \\
\hline VIC2646 & $G R$ & 208 & A2a \\
\hline VIC4055 & $G R$ & 208 & A2a \\
\hline VIC527 & G & 20A_1 & $A_{2}$ \\
\hline VIC61 & 01 & 19A 2 & A1a \\
\hline VICBO3 & G & 20A_1 & $\mathrm{A}_{2}$ \\
\hline VIC951 & 01 & 19A 2 & A1a \\
\hline
\end{tabular}

C

Marker profiles

sample_id $\begin{array}{lllllllllllllllll}241 & 1059 & 3037 & 8782 & 11083 & 14408 & 14805 & 17747 & 17858 & 18060 & 23403 & 25663 & 26144 & 28144 & 28881 & 28882 & 2888\end{array}$

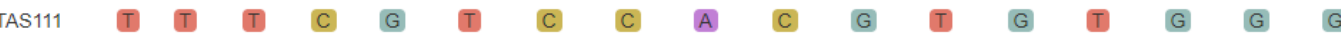
$\begin{array}{llllllllllllllllll}T A S 221 & C & C & C & \mathbb{I} & G & \text { C } & \text { C } & \text { C } & G & \text { I } & A & G & G & \text { C } & G & G & G\end{array}$ $\begin{array}{llllllllllllllllll}\text { TAS25 } & \text { C } & C & C & \text { I } & G & \text { C } & \text { I } & C & A & C & A & G & G & C & G & G & G\end{array}$

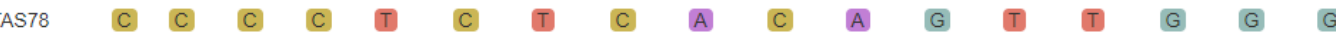

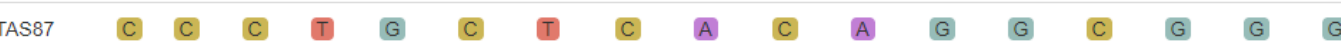
$\begin{array}{llllllllllllllllll}V 1 C 07 & C & C & C & \mathbb{1} & G & C & C & C & A & C & A & G & G & C & G & G & G\end{array}$

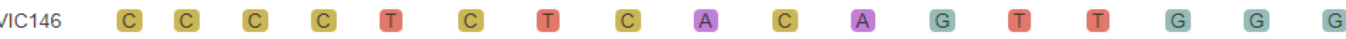

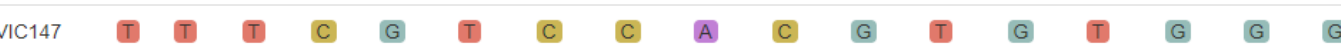
VIC149 II C C VIC1490 C C C C C C C C

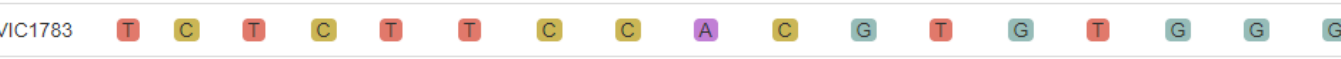

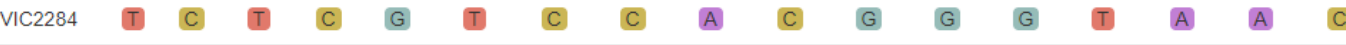

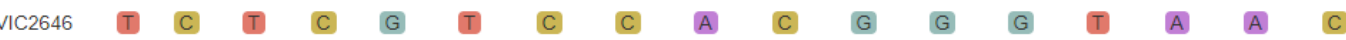

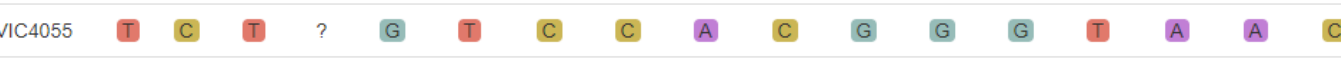
$\begin{array}{llllllllllllllllll}\text { VIC527 } & \text { I } & C & \mathbb{1} & C & G & \text { I } & C & C & A & C & G & G & G & \mathbb{T} & G & G & G\end{array}$

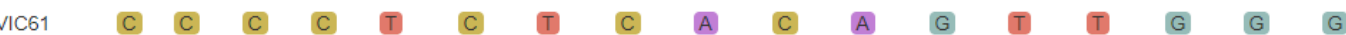

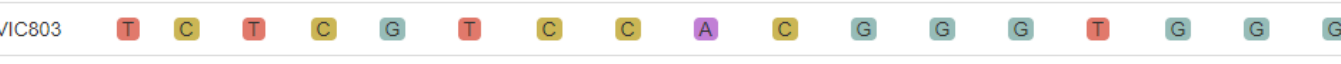

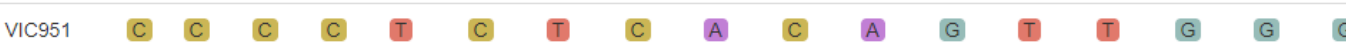

Call Manhattan Scores

GISAID

New Nextstrain

Old Nextstrain

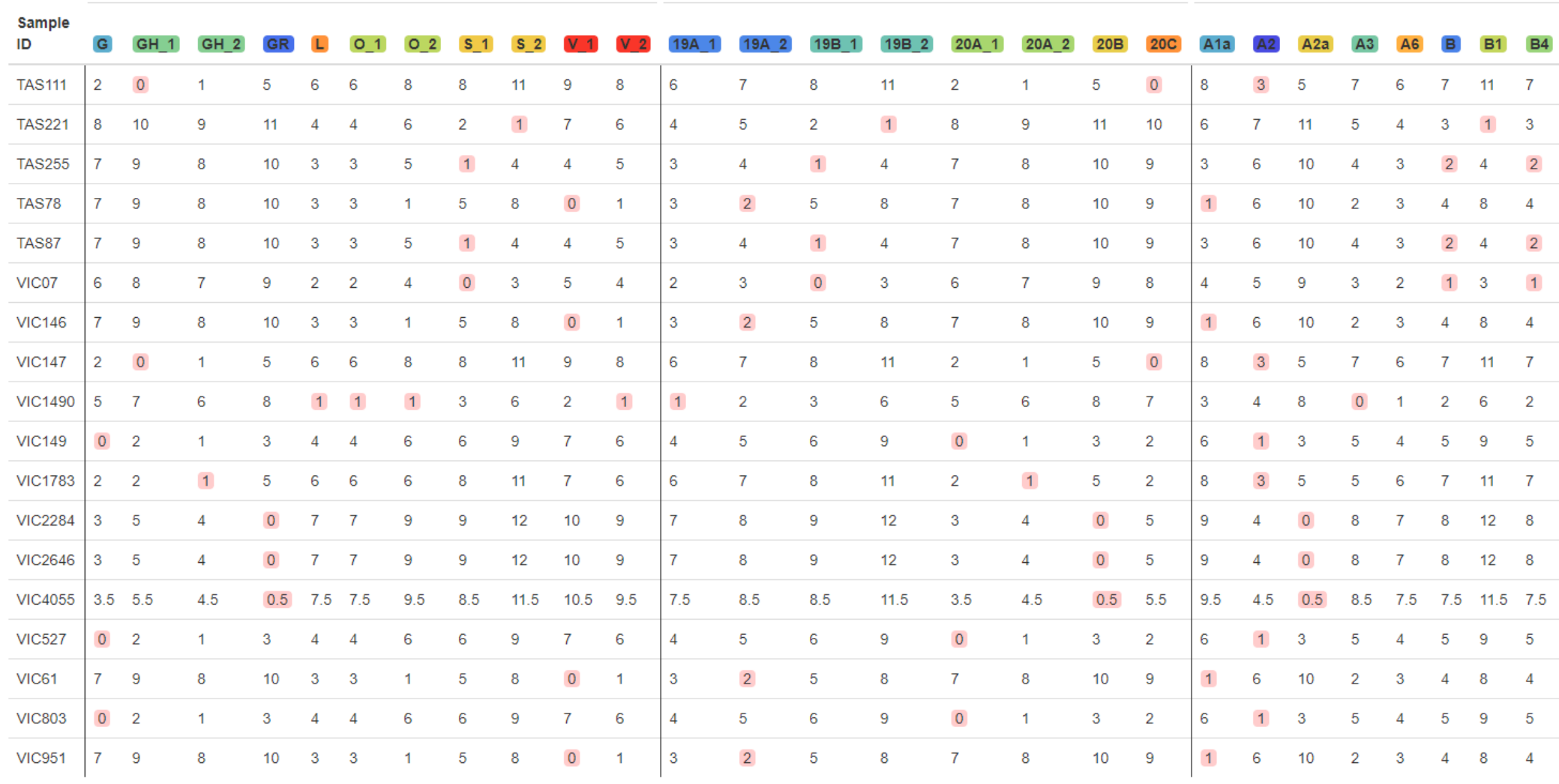

D

\section{Coverages}

$\begin{array}{llllllllllllllllll}\text { sample_id } & 241 & 1059 & 3037 & 8782 & 11083 & 14408 & 14805 & 17747 & 17858 & 18060 & 23403 & 25563 & 26144 & 28144 & 28881 & 28882 & 28883\end{array}$ $\begin{array}{llllllllllllllllll}\text { TAS111 } & 1113 & 3541 & 2281 & 1413 & 2548 & 13151 & 2892 & 2179 & 208 & 4541 & 6081 & 7736 & 7157 & 4404 & 48 & 49 & 48\end{array}$ $\begin{array}{llllllllllllllllll}\text { TAS221 } & 343 & 4061 & 2942 & 20 & 333 & 4051 & 1175 & 1332 & 110 & 4957 & 3842 & 9838 & 2268 & 3382 & 332 & 338 & 338\end{array}$ $\begin{array}{llllllllllllllllll}\text { TAS255 } & 2010 & 3293 & 5381 & 1861 & 5409 & 5565 & 6360 & 1542 & 1084 & 3225 & 5649 & 5850 & 7573 & 4049 & 960 & 976 & 972\end{array}$ $\begin{array}{llllllllllllllllll}\text { TAS78 } & 5139 & 2270 & 4553 & 8301 & 3509 & 5831 & 5181 & 2320 & 2534 & 2714 & 4589 & 3351 & 9394 & 3815 & 325 & 330 & 325\end{array}$ $\begin{array}{llllllllllllllllll}\text { TAS87 } & 5122 & 2840 & 5242 & 9564 & 4017 & 6508 & 5814 & 2538 & 2993 & 2832 & 5984 & 3403 & 9493 & 4324 & 761 & 759 & 750\end{array}$ $\begin{array}{llllllllllllllllll}\text { VIC07 } & 81 & 2351 & 3756 & 889 & 5263 & 8551 & 3419 & 1375 & 868 & 3143 & 7999 & 7547 & 11860 & 5803 & 293 & 293 & 290\end{array}$ $\begin{array}{llllllllllllllllll}\text { VIC146 } & 1134 & 3440 & 3933 & 134 & 4515 & 7436 & 5500 & 1575 & 130 & 2283 & 4195 & 7138 & 15864 & 6117 & 654 & 656 & 643\end{array}$ $\begin{array}{llllllllllllllllll}\text { VIC147 } & 2825 & 2686 & 4042 & 454 & 5476 & 7477 & 5322 & 1270 & 169 & 2531 & 3464 & 5896 & 12948 & 5227 & 456 & 456 & 453\end{array}$ $\begin{array}{llllllllllllllllll}\text { VIC 149 } & 1014 & 1328 & 1587 & 92 & 1889 & 3144 & 2172 & 552 & 38 & 1144 & 1413 & 2826 & 6102 & 2351 & 228 & 230 & 231\end{array}$ $\begin{array}{llllllllllllllllll}\text { VIC1490 } & 3794 & 2768 & 2954 & 2796 & 1772 & 2498 & 2084 & 1396 & 1815 & 1328 & 4722 & 2312 & 211 & 1960 & 1657 & 1656 & 1652\end{array}$ $\begin{array}{llllllllllllllllll}\text { VIC1783 } & 479 & 1276 & 2730 & 644 & 2480 & 1854 & 2571 & 438 & 359 & 1549 & 3731 & 1806 & 3546 & 2655 & 410 & 417 & 408\end{array}$ $\begin{array}{llllllllllllllllll}\text { Vic2284 } & 1638 & 3757 & 4090 & 2696 & 6238 & 6073 & 8218 & 2522 & 2460 & 2994 & 7585 & 7685 & 8515 & 5481 & 2220 & 2218 & 224\end{array}$ $\begin{array}{lllllllllllllllllll}\text { VC2646 } & 8395 & 5293 & 7220 & 2369 & 6930 & 7852 & 9449 & 4811 & 5469 & 4572 & 7063 & 8974 & 14717 & 6028 & 2266 & 2261 & 2295\end{array}$ $\begin{array}{llllllllllllllllll}\text { VIC } 4055 & 11 & 4418 & 5709 & 6 & 144 & 7465 & 18 & 1058 & 22 & 3545 & 1292 & 8002 & 9392 & 683 & 13 & 13 & 13\end{array}$ $\begin{array}{llllllllllllllllll}\text { VIC527 } & 4461 & 2633 & 5538 & 4410 & 4662 & 6503 & 6088 & 1043 & 580 & 2709 & 5345 & 3042 & 8384 & 3781 & 979 & 992 & 983\end{array}$ $\begin{array}{llllllllllllllllll}\text { V1C61 } & 13135 & 9784 & 5006 & 144 & 10806 & 23760 & 6922 & 2384 & 604 & 7179 & 6795 & 8752 & 2558 & 6003 & 48 & 47 & 47\end{array}$ $\begin{array}{llllllllllllllllll}\text { VIC803 } & 3474 & 2584 & 3140 & 46 & 4864 & 6415 & 5543 & 1191 & 641 & 2927 & 6388 & 3440 & 4426 & 4692 & 198 & 199 & 194\end{array}$ 